\title{
Limiting Behavior of Travelling Waves for the Modified Degasperis-Procesi Equation
}

\author{
Jiuli Yin, Liuwei Zhao, and Shanyu Ding \\ Nonlinear Scientific Research Center, Faculty of Science, Jiangsu University, Zhenjiang, Jiangsu 212013, China \\ Correspondence should be addressed to Jiuli Yin; yjl@mail.ujs.edu.cn
}

Received 18 February 2014; Revised 6 May 2014; Accepted 27 May 2014; Published 9 July 2014

Academic Editor: Xing Biao Hu

Copyright ( 2014 Jiuli Yin et al. This is an open access article distributed under the Creative Commons Attribution License, which permits unrestricted use, distribution, and reproduction in any medium, provided the original work is properly cited.

Using an improved qualitative method which combines characteristics of several methods, we classify all travelling wave solutions of the modified Degasperis-Procesi equation in specified regions of the parametric space. Besides some popular exotic solutions including peaked waves, and looped and cusped waves, this equation also admits some very particular waves, such as fractal-like waves, double stumpons, double kinked waves, and butterfly-like waves. The last three types of solutions have not been reported in the literature. Furthermore, we give the limiting behavior of all periodic solutions as the parameters trend to some special values.

\section{Introduction}

The Degasperis-Procesi (DP) equation

$$
u_{t}+c_{0} u_{x}-u_{x x t}+4 u u_{x}=3 u_{x} u_{x x}+u u_{x x x}
$$

was derived as a member of a one-parameter family of asymptotic shallow water approximations to the Euler equations $[1,2]$. Since its discovery, many papers were devoted to the study of (1) (cf. [3-10] and the citations therein).

The DP equation is of interests because of the following two aspects. On one side, (1) is integrable [1]. On another side, the DP equation presents abundant nonlinear phenomena due to the coexistence of nonlinear convections and nonlinear dispersions. Equation (1) admits wave-breaking phenomena and existence of exotic solution including peakons and cuspons [11-15].

To further complement the study of the DP equation, Wazwaz gave and studied the modified Degasperis-Procesi equation $(\mathrm{mDP})[16]$ :

$$
u_{t}+c_{0} u_{x}-u_{x x t}+4 u^{2} u_{x}=3 u_{x} u_{x x}+u u_{x x x} .
$$

It is clear that the nonlinear convection term $u u_{x}$ has been changed to $u^{2} u_{x}$ in (2). Wazwaz employed these modified forms as a vehicle to explore the change in the physical structure of the solution. Many researchers have obtained abundant travelling wave solutions by different methods. Ma et al. [17] applied the auxiliary equation method to obtain some new solitary and traveling wave solutions. Rui et al. [18] obtained abundant traveling wave solutions by the integral bifurcation method. In [19], a new characteristic of solitary wave solutions, bell-shaped solitary wave, and peakon coexisting for the same wave speed in $\mathrm{mDP}$ equation was found by Liu and Ouyang.

It is noted that the nonsmooth wave solutions of the $\mathrm{mDP}$ equation obtained in the previous studies have not been checked in a weak solution way. And new solutions of the $\mathrm{mDP}$ equation have been founded.

Motivated by the above two aspects, we try to answer them. It is noticeable that our method to search solutions of the $\mathrm{mDP}$ equation is combined with some characteristics of several methods [10-15]. Three characters lie in our method. (i) Definition of weak solutions of the $\mathrm{mDP}$ equation is given. (ii) More new exotic solutions are obtained. The parameter space is divided in further detail. (iii) The limiting behavior of traveling wave solutions is given.

This paper is organized as follows. In Section 2, we give the definition of weak solutions. In Section 3, we give 
theorems of the classification of travelling waves in the $\mathrm{mDP}$ equation. In Section 4, we give the proof. Section 5 is the conclusion.

\section{Definitions and Notations}

In this section, we will give the classification of travelling wave solutions of (2), which is stated in Theorem 3.

For a travelling wave $u(x, t)=\phi(x-c t),(2)$ takes the form

$$
-c \phi_{x}+c_{0} \phi_{x}+c \phi_{x x x}+4 \phi^{2} \phi_{x}=3 \phi_{x} \phi_{x x}+\phi \phi_{x x x}
$$

where $c$ is the wave speed.

By integrating with respect to $x$ and letting the integral constant be zero, (3) becomes

$$
\frac{1}{2}\left((\phi-c)^{2}\right)_{x x}=\frac{4}{3} \phi^{2}-c \phi+c_{0} \phi+\alpha .
$$

Equation (4) makes sense for all $\phi \in H_{\text {loc }}^{1}(\mathbb{R})$. The following definition is therefore natural.

Definition 1. A function $\phi \in H_{\text {loc }}^{1}(\mathbb{R})$ is a travelling wave solution of (2) if $\phi$ satisfies (4) in distribution sense for some $\alpha \in \mathbb{R}$.

Like the proof of the proceeding of Lemmas 4 and 5 in [15], we can give the following definition of weak traveling wave solutions.

Definition 2. Any bounded function $\phi$ belongs to $H_{\text {loc }}^{1}(\mathbb{R})$ and is a travelling wave solution of (2) with speed $c$ if it satisfies the following two statements.

(a) There are disjoint open intervals $E_{i}, i \geq 1$, and a closed set $C$ such that $\mathbb{R} \backslash C=\bigcup_{i=1}^{\infty} E_{i}, \phi \in C^{\infty}\left(E_{i}\right)$ for $i \geq 1$, $\phi(x) \neq c$ for $x \in \bigcup_{i=1}^{\infty} E_{i}$, and $\phi(x)=c$ for $x \in C$.

(b) There is an $\alpha \in \mathbb{R}$ such that

(i) For each $\alpha \in \mathbb{R}$, there exists $\beta \in \mathbb{R}$ such that

$$
\phi_{x}^{2}=F(\phi), \quad x \in E_{i},
$$

where

$F(\phi)$

$=\frac{(8 / 15) \varphi^{5}-(2 / 3) c \varphi^{4}+(2 / 3)\left(c_{0}-c\right) \varphi^{3}-\left(c_{0}-c\right) c \varphi^{2}+h}{(\varphi-c)^{2}}$

and $\phi \rightarrow c$, at any finite endpoint of $E_{i}$.

(ii) If $C$ has strictly positive Lebesgue measure $\mu(C)>0$, we have $\alpha=-(1 / 3) c^{2}-c_{0} c$.

\section{Main Results}

Let $h_{m}, h_{n}$, and $h_{c}$ be defined as in (11). All travelling wave solutions $\varphi(x-c t)$ of (1) are smooth except at points where $\phi=c$. We state our main result as follows.
Theorem 3. If $c_{0}<(1 / 16)\left(16 c-75 c^{2}\right)$, any travelling wave solution of (1) falls into one of the following categories.

(1) For $h \leq h_{m}$, there are no bounded traveling wave solutions.

(2) For $h \in\left(h_{m}, h_{c}\right]$, there exists a periodic wave solution.

(3) For $h \in\left(h_{c}, 0\right)$, there exist a periodic wave solution and a looped periodic wave solution. Moreover, as $h \rightarrow 0$, the periodic wave solution converges to a solitary wave solution pointing downward and the looped periodic wave solution converges to a looped wave solution.

(4) For $h \in\left(0, h_{n}\right)$, there exists a looped periodic wave solution. Moreover, the looped periodic wave solution converges to a rotated looped wave solution.

(5) For $h>h_{n}$, there exists a cusped periodic wave solution.

Theorem 4. If $c_{0}<(1 / 16)\left(16 c-75 c^{2}\right)$, any travelling wave solution of (1) falls into one of the following categories.

(1) For $h \leq h_{m}$, there are no bounded traveling wave solutions.

(2) For $h \in\left(h_{m}, h_{c}\right]$, there exists a periodic wave solution.

(3) For $h \in\left(h_{c}, 0\right)$, there exist periodic and looped periodic wave solutions. Moreover, as $h \rightarrow 0$, the periodic wave solution converges to a solitary wave solution pointing downward and the looped periodic wave solution converges to a butterfly-like wave solution.

(4) For $h>0$, there exists a cusped periodic wave solution.

Theorem 5. If $(1 / 16)\left(16 c-75 c^{2}\right)<c_{0}<(1 / 3)\left(3 c-4 c^{2}\right)$, any travelling wave solution of (1) falls into one of the following categories.

(1) For $h \leq h_{m}$, there are no bounded traveling wave solutions.

(2) For $h \in\left(h_{m}, h_{c}\right]$, there exists a periodic wave solution.

(3) For $h \in\left(h_{c}, h_{n}\right)$, there exist a periodic wave solution and a looped periodic wave solution. Moreover, as $h \rightarrow$ $h_{n}$, the looped periodic wave solution converges to a looped wave solution pointing downward.

(4) If $h \in\left(h_{n}, 0\right)$, there exist a periodic wave solution and a cusped periodic wave solution. Moreover, as $h \rightarrow 0$, the periodic wave solution converges to a solitary wave solution pointing downward and the cusped periodic wave solution converges to a cusped wave solution.

(5) If $h>0$, there exists a cusped periodic wave solution.

Theorem 6. If $c_{0}=(1 / 3)\left(3 c-4 c^{2}\right)$, any travelling wave solution of (1) falls into one of the following categories.

(1) For $h \leq h_{m}$, there are no bounded traveling wave solutions.

(2) For $h \in\left(h_{c}, 0\right)$, there exists a periodic wave solution. Moreover, as $h \rightarrow 0$, there exists a cusped wave solution and the periodic wave solution converges to solitary wave solution pointing downward.

(3) For $h>0$, there exists a cusped periodic wave solution. 
Theorem 7. If $(1 / 3)\left(3 c-4 c^{2}\right)<c_{0}<(1 / 5)\left(5 c-2 c^{2}\right)$, any travelling wave solution of (1) falls into one of the following categories.

(1) For $h \leq h_{m}$, there are no bounded traveling wave solutions.

(2) For $h \in\left(h_{m}, h_{n}\right]$, there exists a periodic wave solution.

(3) For $h \in\left(h_{n}, h_{c}\right)$, there exist two distinct periodic wave solutions. Moreover, as $h \rightarrow h_{c}$, one of these periodic wave solutions converges to a peaked periodic wave solution pointing downward.

(4) For $h \in\left(h_{c}, 0\right)$, there exist a periodic wave solution and a cusped periodic wave solution. Moreover, as $h \rightarrow 0$, the periodic wave solution converges a solitary wave solution pointing downward and the cusped periodic wave solution converges to a cusped wave solution.

(5) For $h>0$, there exists a cusped periodic wave solution.

Theorem 8. If $c_{0}=(1 / 5)\left(5 c-2 c^{2}\right)$, any travelling wave solution of (1) falls into one of the following categories.

(1) If $h \leq h_{m}$, there are no bounded traveling solitary wave solutions.

(2) If $h \in\left(h_{m}, h_{n}\right]$, there exists a periodic wave solution.

(3) If $h \in\left(h_{n}, 0\right)$, there are two types of periodic wave solution. Moreover, as $h \rightarrow 0$, the two periodic wave solutions converge to solitary wave solution pointing downward and peaked wave solution.

(4) If $h>0$, there exists a cusped periodic wave solution.

Theorem 9. If $(1 / 5)\left(5 c-2 c^{2}\right)<c_{0}<c$, any travelling wave solution of (1) falls into one of the following categories.

(1) For $h \leq h_{m}$, there are no bounded traveling solitary wave solutions.

(2) For $h \in\left(h_{m}, h_{n}\right]$, there exists a periodic wave solution.

(3) For $h \in\left(h_{n}, 0\right)$, there are two types of periodic wave solution. Moreover, as $h \rightarrow 0$, the two periodic wave solutions converge to solitary wave solution pointing downward and solitary wave solution.

(4) For $h \in\left(0, h_{c}\right)$, there exists a periodic wave solution. Moreover, as $h \rightarrow h_{c}$, the periodic wave solution converges to a peaked periodic wave solution.

(5) For $h>h_{c}$, there exists a cusped periodic wave solution.

Theorem 10. If $c_{0} \geq c$, any travelling wave solution of (1) falls into one of the following categories.

(1) For $h \leq 0$, there are no bounded traveling solitary wave solutions.

(2) For $h \in\left(0, h_{c}\right)$, there exists a periodic wave solution. Moreover, as $h \rightarrow 0$, the periodic wave solution converges to a peaked periodic wave solution.

(3) For $h>h_{c}$, there exists a cusped periodic wave solution.
Theorem 11 (composite waves). A countable number of cusped, peaked, and looped waves in the above cases corresponding to the same value of $\alpha$ can be joined at points where $\phi=c$ to form composite waves. If $\mu\left(\phi^{-1}(c)\right)=0$, one can get travelling wave solution with very strange profiles, such as the travelling waves with a fractal appearance (see Figure 1( $k)$ ). For $\alpha=-(1 / 3) c^{2}-c_{0} c$., the composite waves are solutions of (2) even if $\mu\left(\phi^{-1}(c)\right)>0$. Hence we can obtain double stumpons which contain intervals where $\phi=c$ (see Figure $1(l)$ ).

\section{Proof of Theorem 3}

In this section, we will show that the functions satisfying (a) and (b) in Definition 2 consist exactly of the waves stated in Theorem 3.

Let $\phi$ be a function satisfying (a) and (b) and each wave segment solves the equation

$$
\begin{aligned}
\phi_{x}^{2}=F(\phi), \quad x \in E, \\
F(\phi)=\left(\frac{8}{15} \varphi^{5}-\frac{2}{3} c \varphi^{4}+\frac{2}{3}\left(c_{0}-c\right) \varphi^{3}\right. \\
\left.\quad-\left(c_{0}-c\right) c \varphi^{2}+h\right)\left((\varphi-c)^{2}\right)^{-1},
\end{aligned}
$$

for some interval $E$ and constants $c_{0}, h$.

For determining the solutions of (3), we should give the following facts.

Lemma 12. The qualitative behavior of solutions of $\phi_{x}^{2}=F(\phi)$ near points where $F$ has a zero or a pole is as follows.

(1) If $F(\phi)$ has a simple zero at $\phi=m$, the solution $\phi$ to (6) satisfies $\phi(x) \sim m+\lambda\left(\left(x-x_{0}\right)^{2}\right)$, where $\phi\left(x_{0}\right)=\phi_{0}$. It is easy to find that smooth solutions exist if $F(\phi)$ has two simple zeros.

(2) If $F(\phi)$ has a double zero at $\phi=m$, the solution $\phi$ of (6) satisfies $\phi(x) \sim m+\eta \exp (-|x|)$ as $x \rightarrow \infty$. It is easy to find that smooth solitary wave solutions exist if $F(\phi)$ has a simple zero.

(3) Assume that $F(\phi)$ has a double pole at $\phi=m$; then we obtain

$\phi_{x}(x)= \begin{cases}\frac{2}{3} \eta\left|x-x_{0}\right|^{-1 / 3}+o\left((\phi-m)^{1 / 3}\right), & x \downarrow x_{0}, \\ -\frac{2}{3} \eta\left|x-x_{0}\right|^{-1 / 3}+o\left((\phi-m)^{1 / 3}\right), & x \uparrow x_{0},\end{cases}$

where $\phi\left(x_{0}\right)=c$ and $\eta$ is some constant. Particularly, if $c=\max _{x \in R} \phi(x)$ (or $\min _{x \in R} \phi(x)$ ), we get $\lim _{x \rightarrow x_{0}} \phi_{x}(x)=-\lim _{x \leftarrow x_{0}} \phi_{x}(x)= \pm \infty$. Then the function $\phi$ is called a cusped wave solution. If $c \in\left(\min _{x \in R} \phi(x), \max _{x \in R} \phi(x)\right)$, then the function $\phi$ is called a looped wave.

(4) Peaked waves occur when the evolution of $\phi$ according to (6) suddenly changes direction at $\varphi=c$ where $F(c) \neq 0$ and $\varphi\left(x_{0}\right)=c$; that is, $\lim _{x \rightarrow x_{0}} \phi_{x}(x)=$ $-\lim _{x \leftarrow x_{0}} \phi_{x}(x) \neq \pm \infty$. 


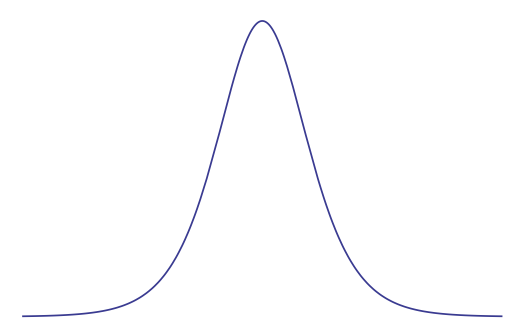

(a) Solitary wave

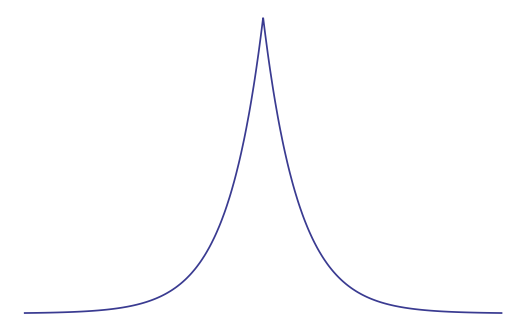

(c) Peaked wave

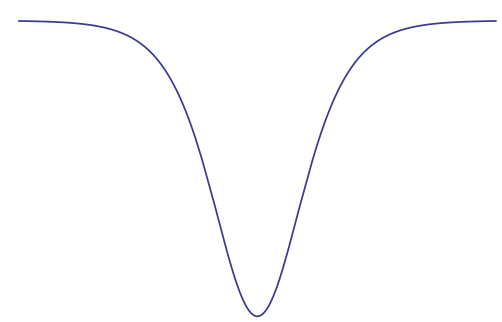

(b) Solitary wave pointing downwards

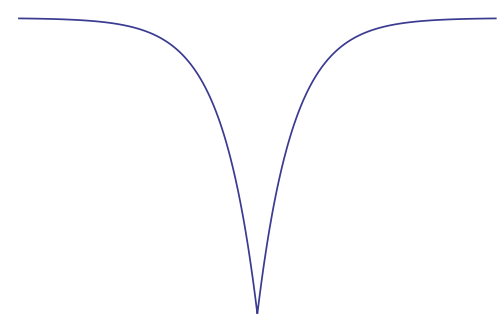

(d) Peaked wave pointing downwards

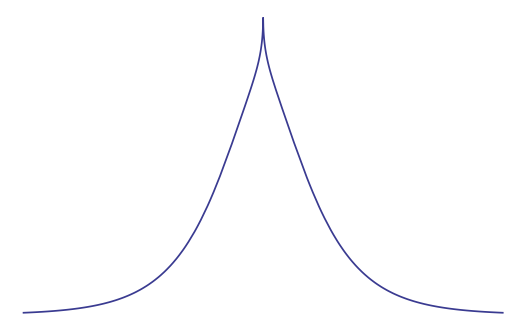

(e) Cusped wave

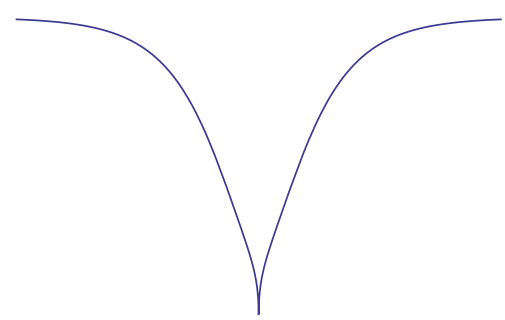

(f) Cusped wave pointing downwards

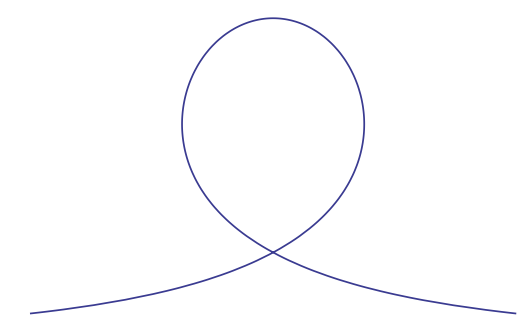

(g) Looped wave

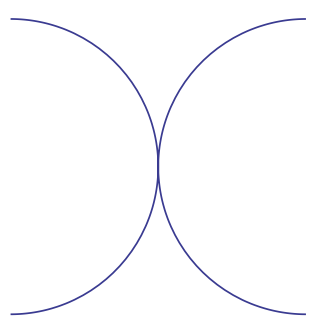

(j) Butterfly-like waves

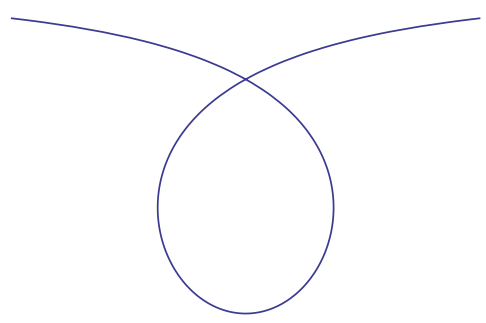

(h) Rotated looped wave

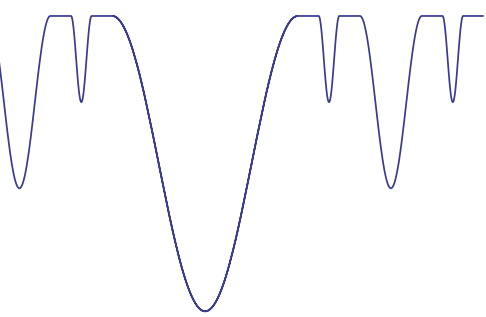

(k) Fractal-like wave

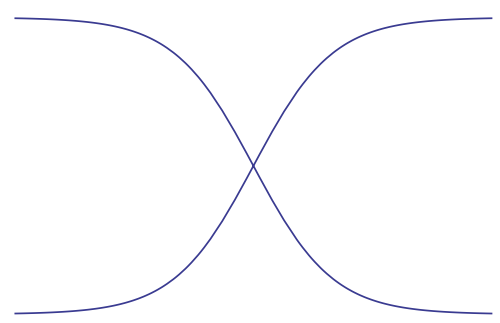

(i) Double kinked wave

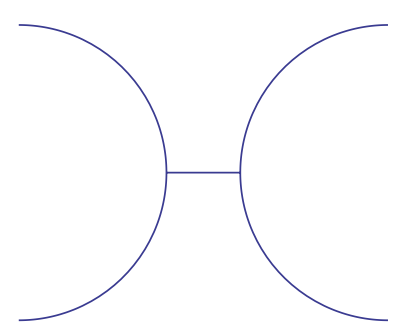

(1) Double stumpon

FIgURE 1: Travelling waves in (2) as $t=0$.

(5) Double kinked waves occur when the right-hand side of (6) has two double zeros which are not opposite numbers, and $\varphi=c$ is not between the two zeros. When the pole $\varphi=c$ falls in the interval of the two zeros, butterfly-like waves occur. One reason for the occurrence of these two new solutions is that the solutions to (2) must be symmetry because this equation is invariable under the transformation $x \rightarrow-x$.
Now we apply the above analysis to

$$
\begin{aligned}
\phi_{x}^{2}(x)= & Y(\phi) \\
= & \left(\frac{8}{15} \phi^{5}-\frac{2}{3} c \phi^{4}+\frac{2}{3}\left(c_{0}-c\right) \phi^{3}\right. \\
& \left.\quad-\left(c_{0}-c\right) c \phi^{2}+h\right)\left((\phi-c)^{2}\right)^{-1} .
\end{aligned}
$$




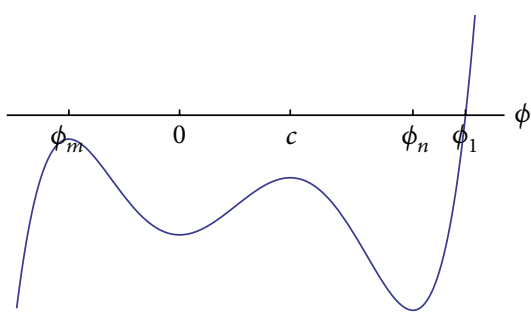

(a) $h<h_{m}$

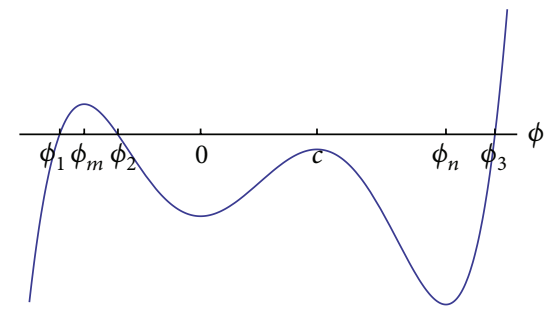

(c) $h \in\left(h_{m}, h_{c}\right)$

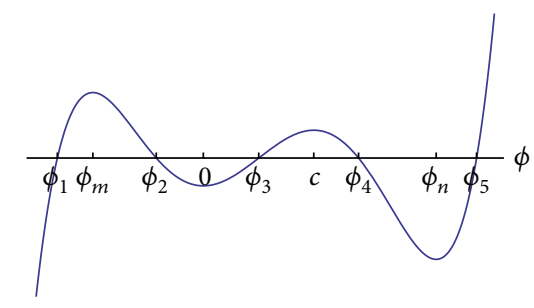

(e) $h \in\left(h_{c}, 0\right)$

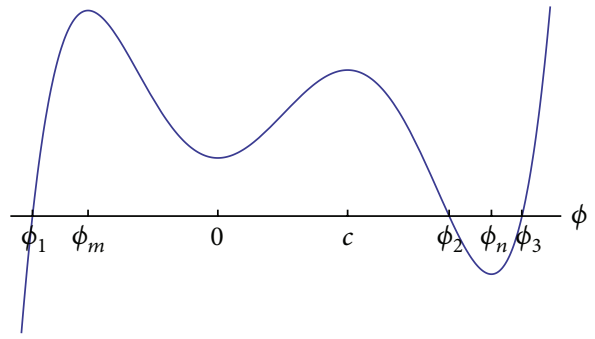

(g) $h \in\left(0, h_{n}\right)$

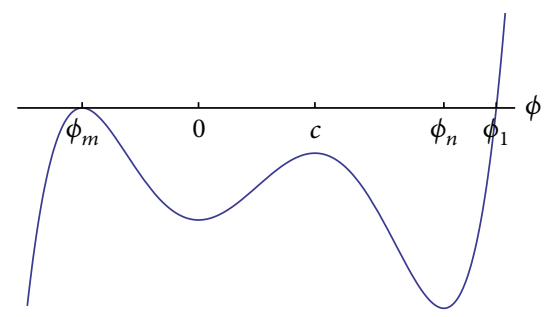

(b) $h \rightarrow h_{m}$

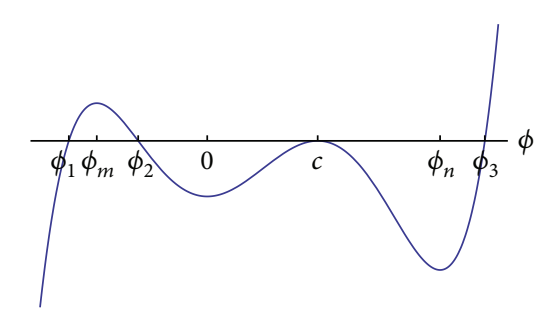

(d) $h \rightarrow h_{c}$

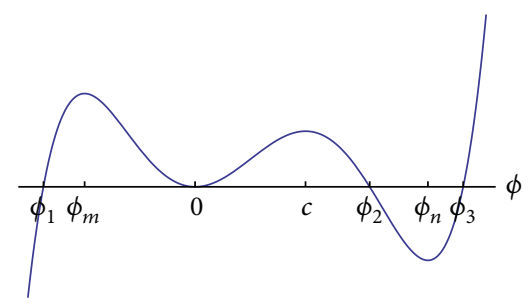

(f) $h \rightarrow 0$

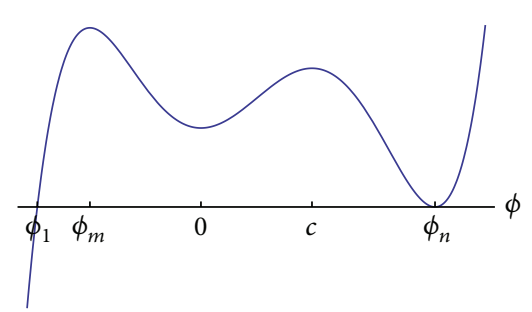

(h) $h \rightarrow h_{n}$

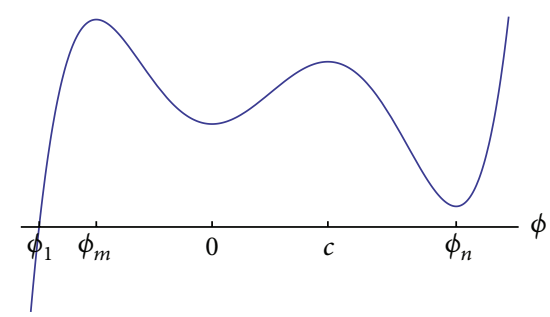

(i) $h>h_{n}$

Figure 2: The graph of $F(\phi)$ as $c_{0}<(1 / 16)\left(16 c-75 c^{2}\right)$.

For convenience, we define $F(\phi), P(\phi)$, and $G(\phi)$ where

If $c>c_{0}$, we define

$$
\begin{gathered}
F(\phi)=\frac{8}{15} \phi^{5}-\frac{2}{3} c \phi^{4}+\frac{2}{3}\left(c_{0}-c\right) \phi^{3}-\left(c_{0}-c\right) c \phi^{2}+h, \\
P(\phi)=\frac{8}{15} \phi^{5}-\frac{2}{3} c \phi^{4}+\frac{2}{3}\left(c_{0}-c\right) \phi^{3}-\left(c_{0}-c\right) c \phi^{2}, \\
P^{\prime}(\phi)=\frac{2}{3} \phi(\phi-c)\left(4 \phi^{2}-3 c+3 c_{0}\right) .
\end{gathered}
$$

$$
\begin{gathered}
\phi_{m}=-\frac{\sqrt{3 c-3 c_{0}}}{2}, \\
\phi_{n}=\frac{\sqrt{3 c-3 c_{0}}}{2} .
\end{gathered}
$$




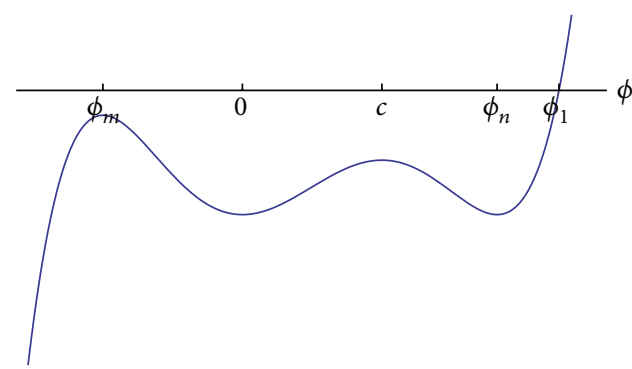

(a) $h<h_{m}$

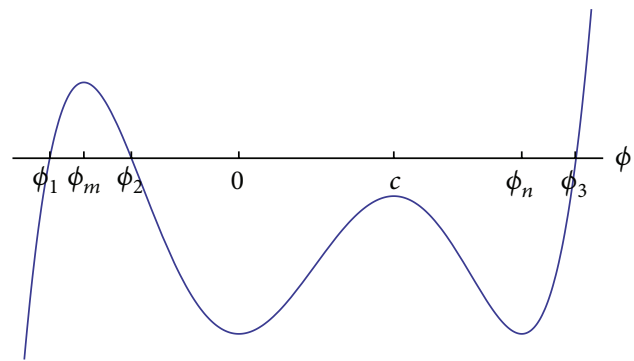

(c) $h \in\left(h_{m}, h_{c}\right)$

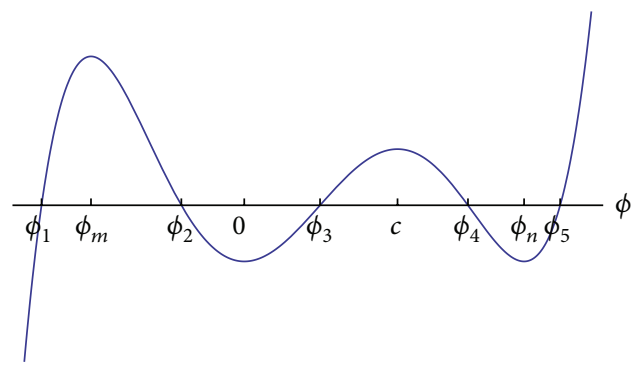

(e) $h \in\left(h_{c}, 0\right)$

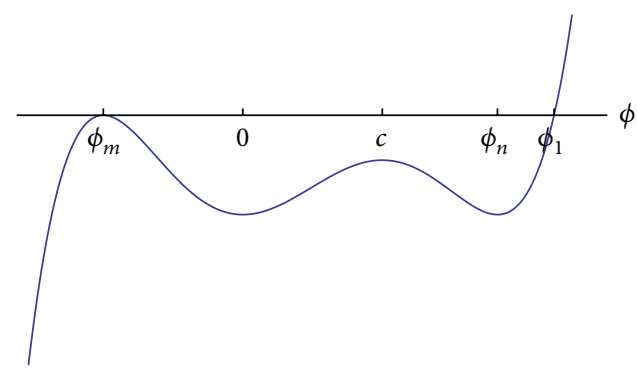

(b) $h \rightarrow h_{m}$

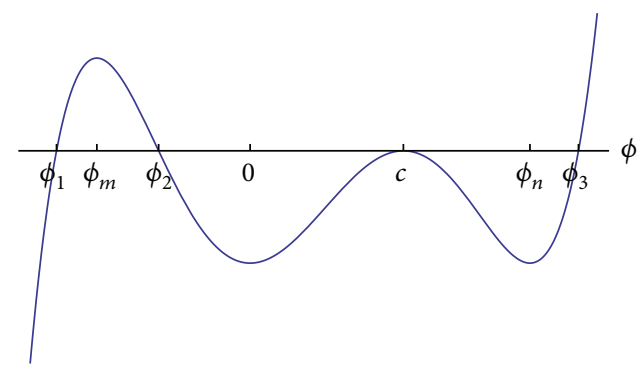

(d) $h \rightarrow h_{c}$

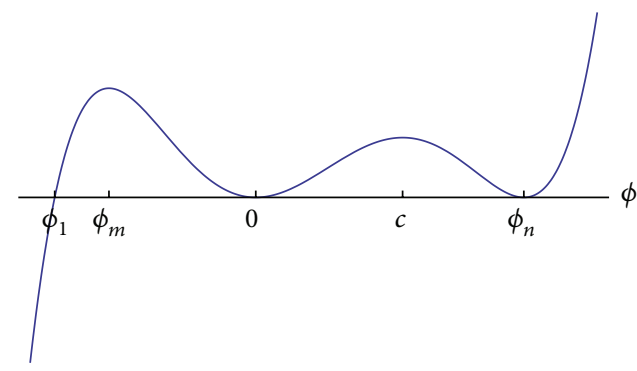

(f) $h \rightarrow 0$

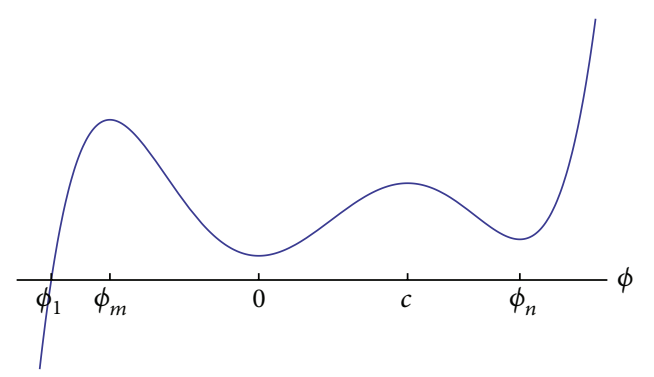

(g) $h>0$

Figure 3: The graph of $F(\phi)$ as $c_{0}=(1 / 16)\left(16 c-75 c^{2}\right)$.

Let

$$
\begin{gathered}
h_{m}=-P\left(\phi_{m}\right), \quad h_{n}=-P\left(\phi_{n}\right), \\
h_{c}=-P(c) .
\end{gathered}
$$

Remark 13. It is not difficult to find that $Y(\phi)$ has the same zero points as $F(\phi)$ except $\phi=c$. It is easy to see that a change $h$ in equation (6) will shift the graph vertically up or down.

Now we consider the existence of solutions and their limiting behavior, which have different analytical forms depending on the values of $c_{0}$ and $h$.
Case $A\left(c_{0}<(1 / 16)\left(16 c-75 c^{2}\right)\right)$. In this case, we have the following.

(1) If $h<h_{m}$, we can get that $F(\phi)$ has only a simple zero $\phi_{1}$ and $F(c)<0$ (see Figure 2(a)). Hence, there are no bounded traveling wave solutions. As $h \rightarrow h_{m}$, it is easy to find that, as $F\left(\phi_{m}\right) \rightarrow 0$ (see Figure 2(b)), $F(\phi)$ has a double zero and a simple zero, so there are no bounded traveling wave solutions either.

(2) If $h \in\left(h_{m}, h_{c}\right)$, we can observe that $F(\phi)$ has three distinct simple zeros denoted by $\phi_{1}, \phi_{2}$, and $\phi_{3}$, and $F(\phi)>$ 0 for $\phi_{1}<\phi<\phi_{2}<c$ (see Figure 2(c)). Then there exists a periodic wave solution $\phi$ with $\phi_{1}=\min _{x \in R} \phi(x)$ 


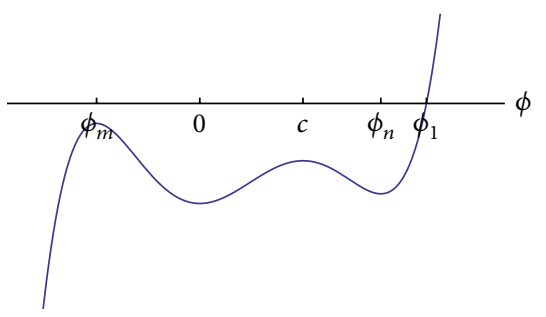

(a) $h<h_{m}$

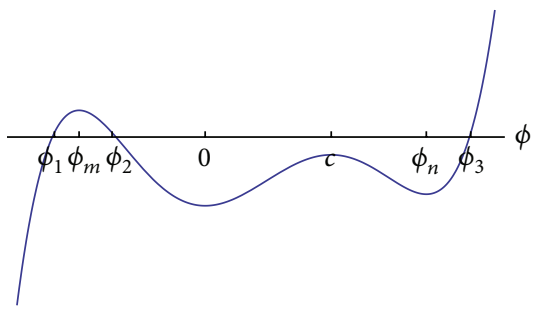

(c) $h \in\left(h_{m}, h_{c}\right)$

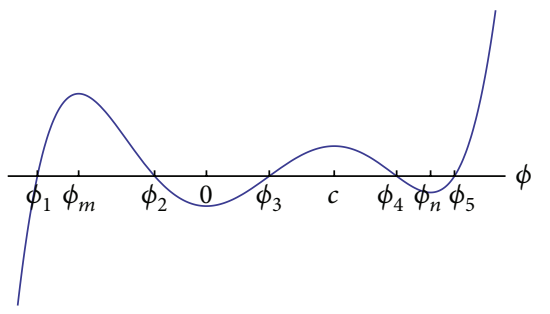

(e) $h \in\left(h_{c}, h_{n}\right)$

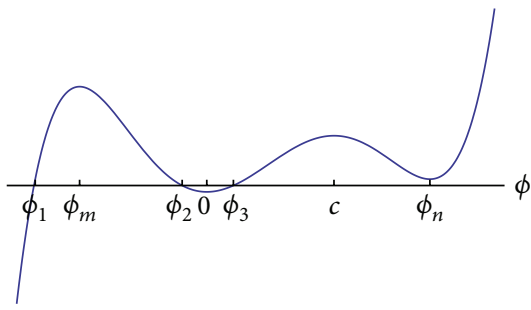

(g) $h \in\left(h_{n}, 0\right)$

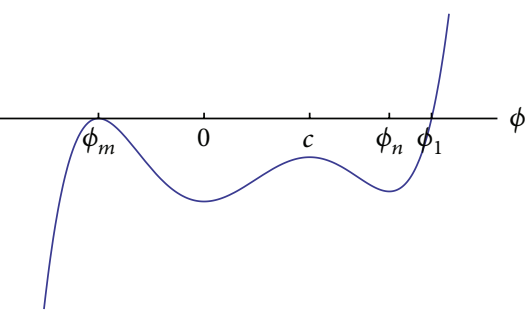

(b) $h \rightarrow h_{m}$

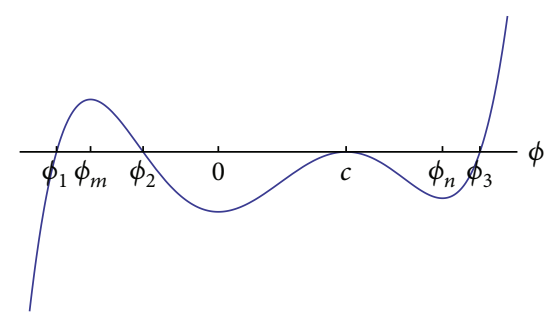

(d) $h \rightarrow h_{c}$

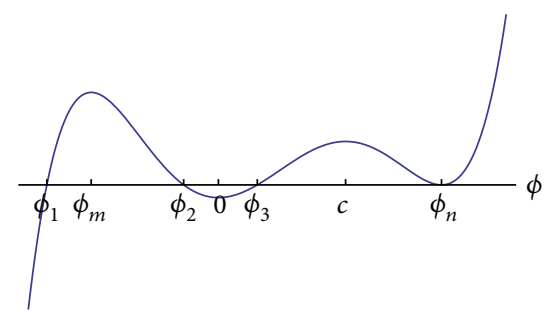

(f) $h \rightarrow h_{n}$

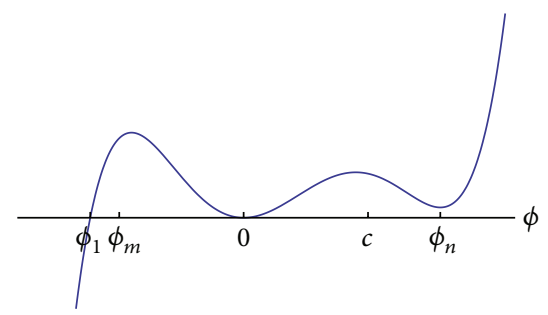

(h) $h \rightarrow 0$

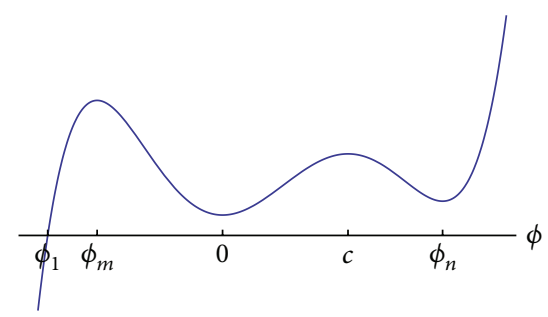

(i) $h>0$

FIGURE 4: The graph of $F(\phi)$ as $(1 / 16)\left(16 c-75 c^{2}\right)<c_{0}<(1 / 3)\left(3 c-4 c^{2}\right)$.

and $\phi_{2}=\max _{x \in R} \phi(x)$. As $h \rightarrow h_{c}$, we have $F(c) \rightarrow 0$ and $F(\phi)$ has three distinct simple zeros denoted by $\phi_{1}, \phi_{2}$, and $\phi_{3}$ (see Figure 2(d)). Then there exists a periodic wave solution $\phi$ with $\phi_{1}=\min _{x \in R} \phi(x)$ and $\phi_{2}=\max _{x \in R} \phi(x)$.

(3) If $h \in\left(h_{c}, 0\right)$, we can deduce that $F(\phi)$ has five distinct simple zeros denoted by $\phi_{1}, \phi_{2}, \phi_{3}, \phi_{4}$, and $\phi_{5}$ (see Figure 2(e)). Obviously, $F(\phi)>0$ for $\phi_{1}<\phi<\phi_{2}<c$. Hence there exists a periodic wave solution $\phi$ with $\phi_{1}=\min _{x \in R} \phi(x)$ and $\phi_{2}=\max _{x \in R} \phi(x)$. Then, we will study the limiting behavior of the periodic wave solution. As $h \rightarrow 0, F(\phi)$ has a double zero at $\phi=0$ and three simple zeros, and $F(\phi)>0$ for $\phi_{1}<\phi<0<c$ (see Figure 2(f)). Hence we deduce that, as $h \rightarrow 0$, the periodic wave solution $\phi$ converges to a solitary wave solution pointing downward with $\phi_{1}=\min _{x \in R} \phi(x)$, $0=\max _{x \in R} \phi(x)$, and $\phi \rightarrow 0$ as $x \rightarrow \infty$. Moreover, if $h \in\left(h_{c}, 0\right)$, we can get $\phi_{3}<c<\phi_{4}$ and $F(\phi)>0$ 


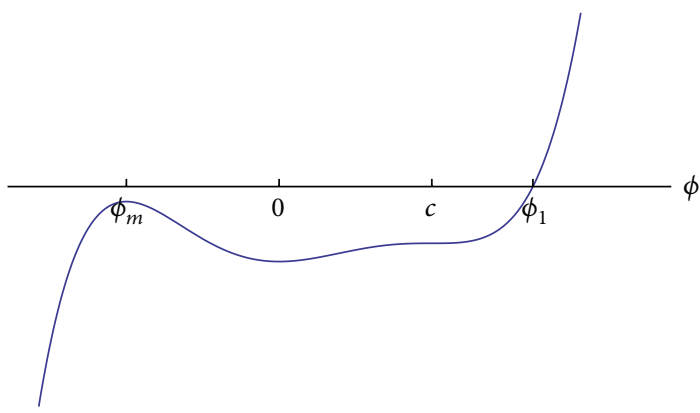

(a) $h<h_{m}$

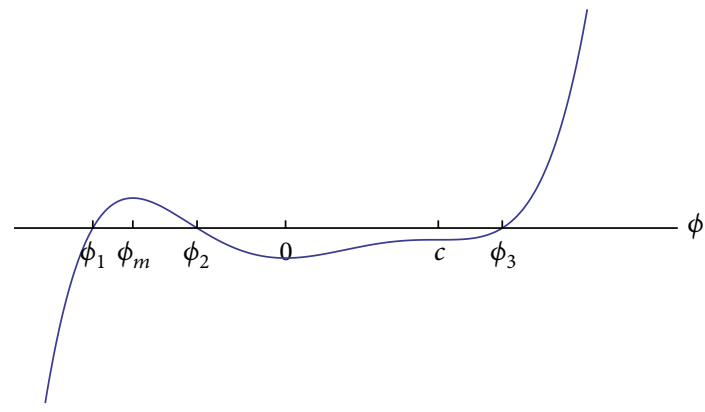

(c) $h \in\left(h_{c}, 0\right)$

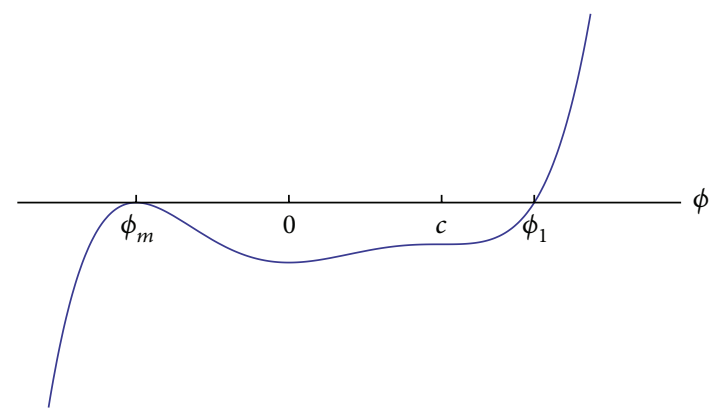

(b) $h \rightarrow h_{m}$

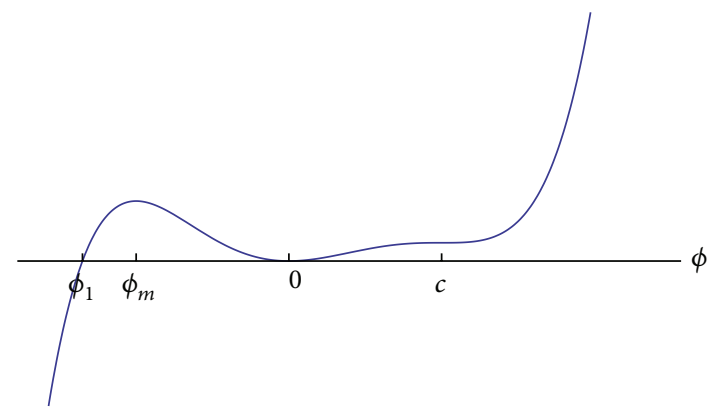

(d) $h \rightarrow 0$

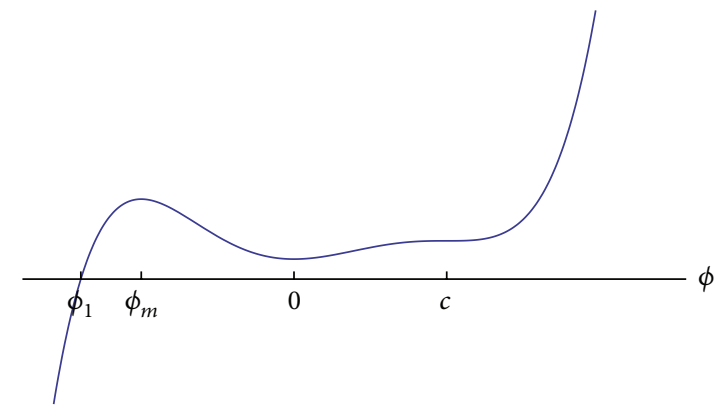

(e) $h>0$

FIGURE 5: The graph of $F(\phi)$ as $c_{0}=(1 / 3)\left(3 c-4 c^{2}\right)$.

for $\phi_{3}<\phi<\phi_{4}$ (see Figure 2(e)). It looks like there exists another periodic wave solution, but we cannot ignore the fact that $\phi_{3}<c<\phi_{4}$. When $\phi$ near $c$, according to Lemma 12, we have $\phi_{x}(x) \sim \lambda\left|x-x_{0}\right|^{-1 / 3}$ and $\phi(x) \sim \eta\left|x-x_{0}\right|$. Hence $\phi_{x}(x) \rightarrow \infty$ as $\phi \rightarrow c$ and the loop is formed. So there exists a looped periodic wave solution $\phi$ with $\phi_{3}=\min _{x \in R} \phi(x)$ and $\phi_{4}=\max _{x \in R} \phi(x)$. Similarly, as $h \rightarrow 0$ (see Figure 2(f)), the looped periodic wave solution converges to a looped wave solution $\phi$ with $0=\min _{x \in R} \phi(x), \phi_{2}=\max _{x \in R} \phi(x)$, and $\phi \rightarrow 0$ as $x \rightarrow \infty$.

(4) If $h \in\left(0, h_{n}\right), F(\phi)$ has three simple zeros, denoted by $\phi_{1}, \phi_{2}$, and $\phi_{3}$, and $F(\phi)>0$ for $\phi_{1}<\phi<\phi_{2}$. Hence there exists a periodic looped wave solution $\phi$ of solution of (3) with $\phi_{1}=\min _{x \in R} \phi(x)$ and $\phi_{2}=\max _{x \in R} \phi(x)$. As $h \rightarrow h_{n}, F(\phi)$ has a simple zero besides $F\left(\phi_{n}\right) \rightarrow 0$ (see Figure $2(\mathrm{~h})$ ). Hence the periodic looped wave solution converges to a looped wave solution $\phi_{1}=\min _{x \in R} \phi(x), \phi_{n}=\max _{x \in R} \phi(x)$, and $\phi \rightarrow \phi_{n}$ as $x \rightarrow \infty$.
(5) If $h>h_{n}$, we can conclude that $F(\phi)$ has only a simple zero, denoted by $\phi_{1}$, and $F(\phi)>0$ for $\phi_{1}<\phi<c$ (see Figure 2(i)). When $\phi$ reaches the pole point $\phi=c$, it will make a sudden turn at this point and yield a cusp at $\phi=c$; that is, $\lim _{x \uparrow x_{0}} \phi_{x}(x)=-\lim _{x \downarrow x_{0}}=\infty$. So there exists a cusped periodic wave solution of $\phi$ with $\phi_{1}=\min _{x \in R} \phi(x)$ and $c=\max _{x \in R} \phi(x)$.

Case $B\left(c_{0}=(1 / 16)\left(16 c-75 c^{2}\right)\right)$. (1) If $h \leq h_{m}$ (see Figures $3(\mathrm{a})$ and $3(\mathrm{~b}))$, similarly to the analysis in Case A, we obtain that there are no bounded traveling solitary wave solutions of (3).

(2) If $h \in\left(h_{m}, h_{c}\right]$ (see Figures 3(c) and 3(d)), there exists a periodic wave solution $\phi$ of (3) with $\phi_{1}=\min _{x \in R} \phi(x)$ and $\phi_{2}=\max _{x \in R} \phi(x)$.

(3) If $h \in\left(h_{c}, 0\right)$, we can deduce that $F(\phi)$ has five distinct simple zeros denoted by $\phi_{1}, \phi_{2}, \phi_{3}, \phi_{4}$, and $\phi_{5}$ (see Figure 3(e)). Obviously, $F(\phi)>0$ for $\phi_{1}<\phi<\phi_{2}<c$. Hence 


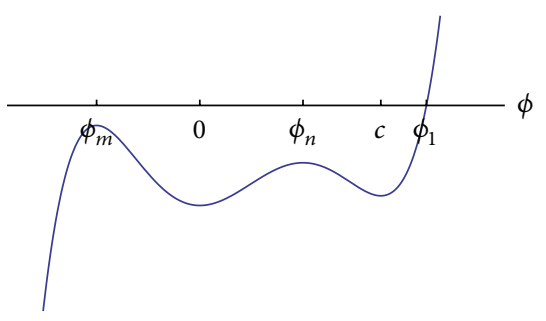

(a) $h<h_{m}$

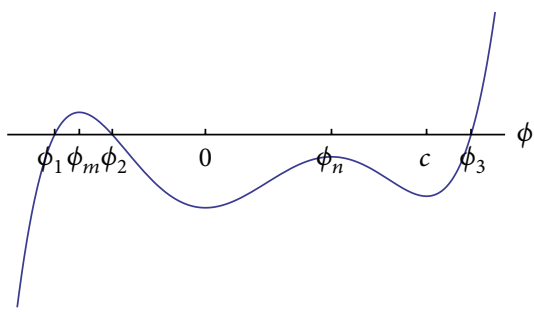

(c) $h \in\left(h_{m}, h_{n}\right)$

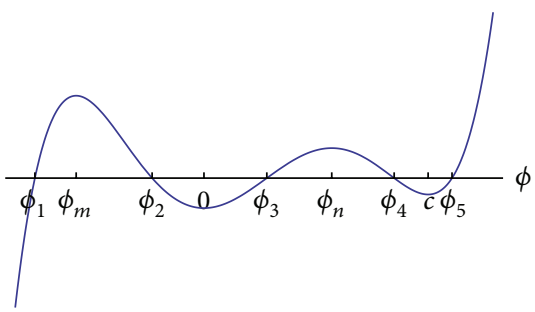

(e) $h \in\left(h_{n}, h_{c}\right)$

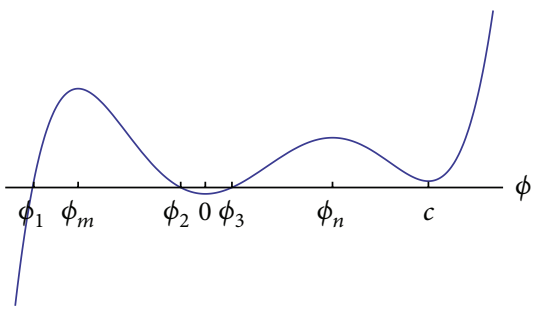

(g) $h \in\left(h_{c}, 0\right)$

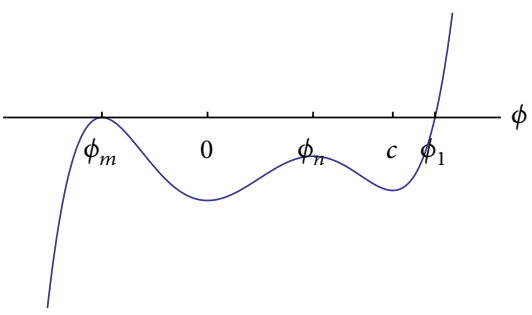

(b) $h \rightarrow h_{m}$

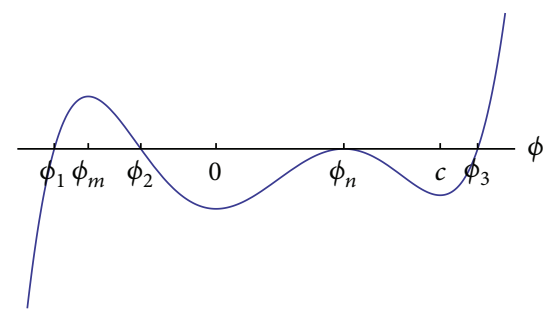

(d) $h \rightarrow h_{n}$

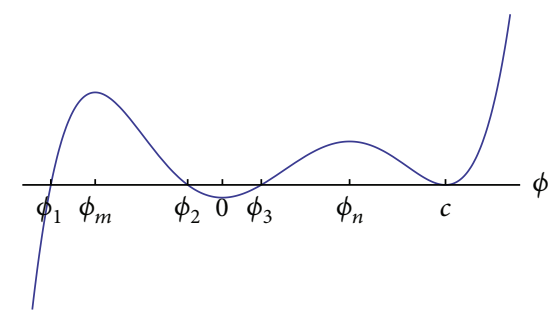

(f) $h \rightarrow h_{c}$

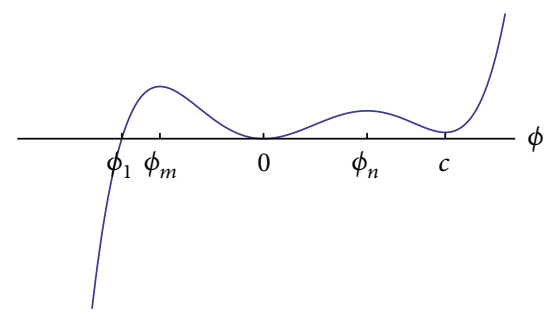

(h) $h \rightarrow 0$

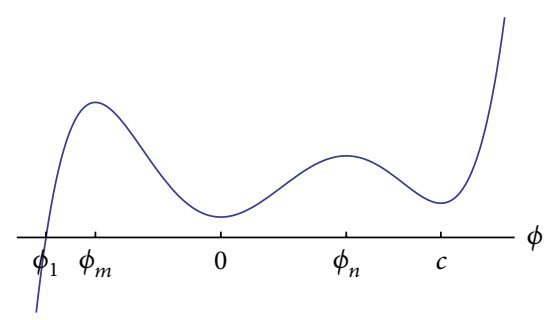

(i) $h>0$

Figure 6: The graph of $F(\phi)$ as $(1 / 3)\left(3 c-4 c^{2}\right)<c_{0}<(1 / 5)\left(5 c-2 c^{2}\right)$.

there exists a periodic wave solution. Moreover, as $h \rightarrow 0$, the periodic wave solution converges to a solitary wave solution pointing downward. Similarly, if $h \in\left(h_{c}, 0\right)$, we can get $\phi_{3}<$ $c<\phi_{4}$ and $F(\phi)>0$ for $\phi_{3}<\phi<\phi_{4}$ (see Figure 3(e)). So there exists a periodic looped wave of (3). Moreover, as $h \rightarrow 0$ (see Figure 3(e)), it looks like that the periodic looped wave solution converges to a looped solitary wave solution. However it is not true due to the fact that both $F(0)$ and $F\left(\phi_{n}\right)$ converge to zero as $h \rightarrow 0$ besides $0<c<\phi_{n}$. According to Lemma 12, there exists a butterfly-like wave solution $\phi$ of (6) with $0=\min _{x \in R} \phi(x)$ and $\phi_{n}=\max _{x \in R} \phi(x)$.

(4) If $h>0$, it is easy to observe that $F(\phi)$ has only one simple zero and $F(c)>0$; hence there exists a cusped periodic wave solution.

Case $C\left((1 / 16)\left(16 c-75 c^{2}\right)<c_{0}<(1 / 3)\left(3 c-4 c^{2}\right)\right)$. In this case, the geometric analysis of $F(\varphi)$ is shown in Figure 4. 


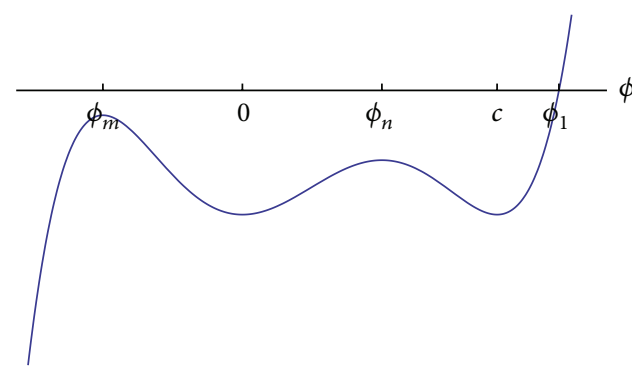

(a) $h<h_{m}$

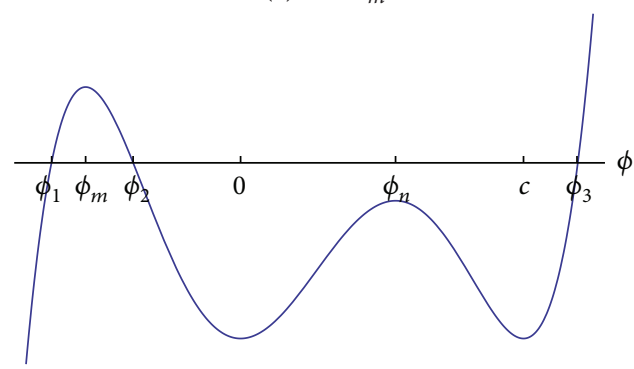

(c) $h \in\left(h_{m}, h_{n}\right)$

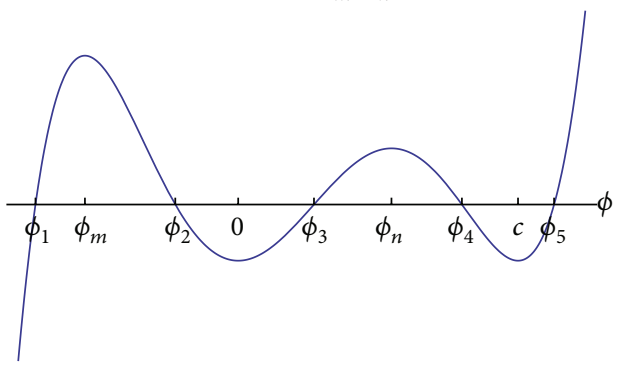

(e) $h \in\left(h_{n}, 0\right)$

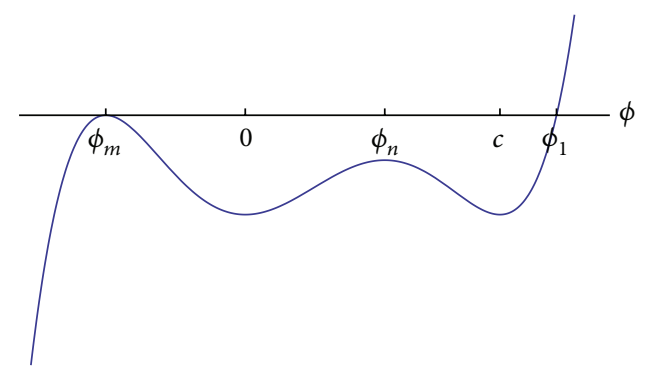

(b) $h \rightarrow h_{m}$

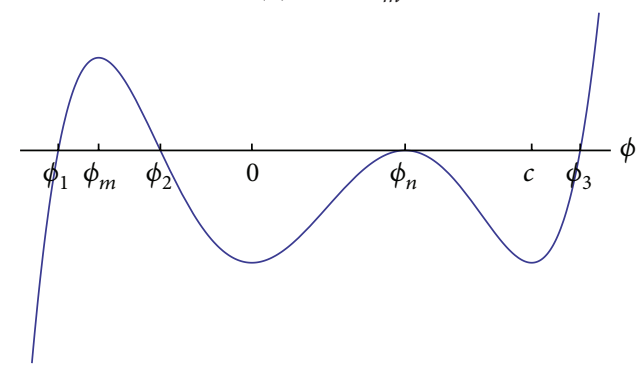

(d) $h \rightarrow h_{n}$

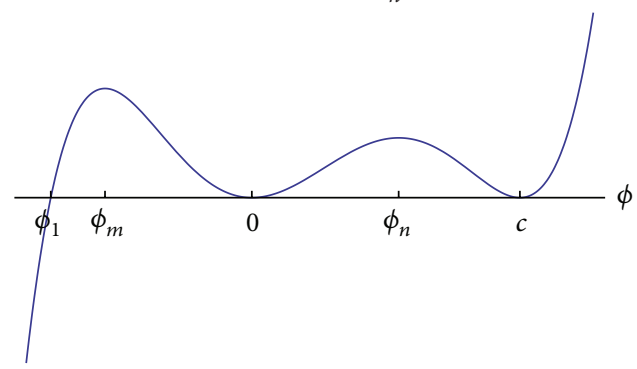

(f) $h \rightarrow 0$

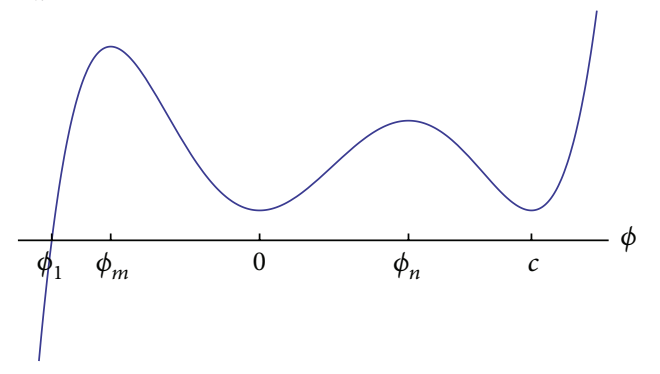

(g) $h>0$

FIGURE 7: The graph of $F(\phi)$ as $c_{0}=(1 / 5)\left(5 c-2 c^{2}\right)$.

The result given in Theorem 5 can be proved in a way similar to that in Case B.

Case $D\left(c_{0}=(1 / 3)\left(3 c-4 c^{2}\right)\right)$. In this case, the geometric analysis of $F(\varphi)$ is shown in Figure 5. The result given in Theorem 6 can be proved in a way similar to that in Case B.

Case $E\left((1 / 3)\left(3 c-4 c^{2}\right)<c_{0}<(1 / 5)\left(5 c-2 c^{2}\right)\right)$. In this case, the geometric analysis of $F(\varphi)$ is shown in Figure 6. The result given in Theorem 7 can be proved in a way similar to that in Case B.

Case $F\left(c_{0}=(1 / 5)\left(5 c-2 c^{2}\right)\right)$. In this case, the geometric analysis of $F(\varphi)$ is shown in Figure 7. The result given in Theorem 8 can be proved in a way similar to that in Case B.
Case $G\left((1 / 5)\left(5 c-2 c^{2}\right)<c_{0}<c\right)$. In this case, the geometric analysis of $F(\varphi)$ is shown in Figure 8. The result given in Theorem 9 can be proved in a way similar to that in Case B.

Case $H\left(c_{0} \geq c\right)$. In this case, the geometric analysis of $F(\varphi)$ is shown in Figure 9. The result given in Theorem 3 can be proved in a way similar to that in Case B.

Then, we will study the existence of composite waves. By Theorems 3-10, any countable number of travelling waves in the above cases corresponding to the same value of $\alpha$ can be joined at points where $\phi=c$ to form composite waves. If $C=\mu\left(\phi^{-1}(c)\right)=0$, then the composite wave is a solution of (2). For $\alpha=-(1 / 3) c^{2}-c_{0} c$, the composite waves are solutions of (2) even if $\mu\left(\varphi^{-1}(c)\right)>0$. Consequently, we can obtain 


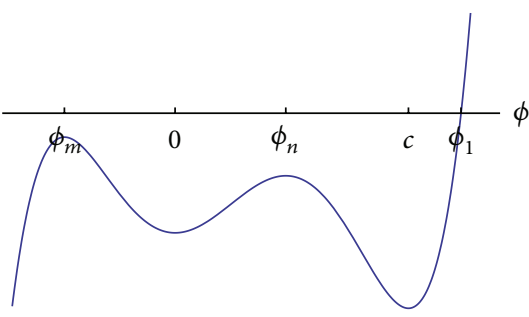

(a) $h<h_{m}$

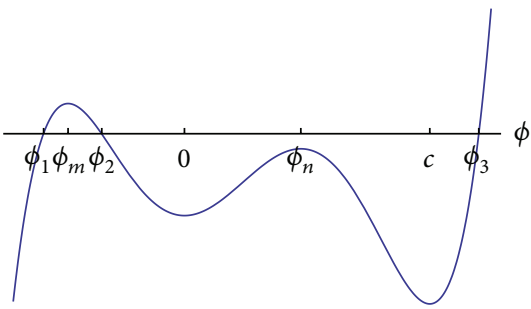

(c) $h \in\left(h_{m}, h_{n}\right)$

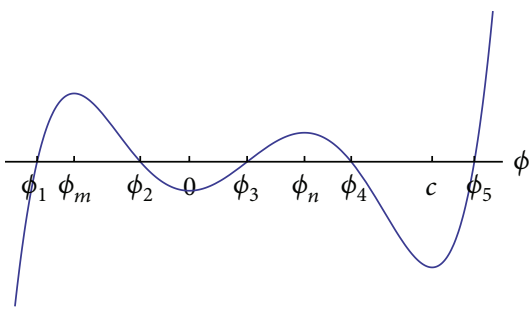

(e) $h \in\left(h_{n}, 0\right)$

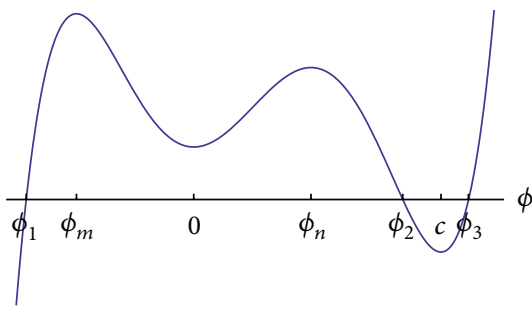

(g) $h \in\left(0, h_{c}\right)$

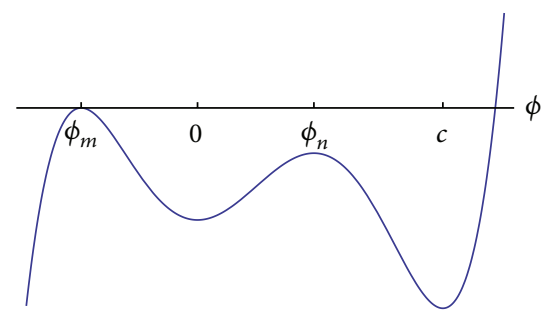

(b) $h \rightarrow h_{m}$

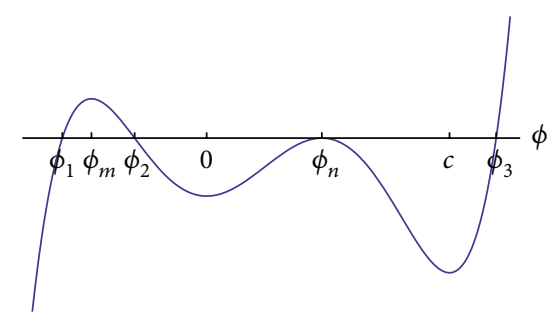

(d) $h \rightarrow h_{n}$

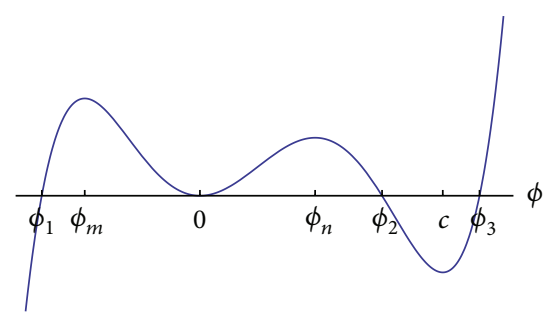

(f) $h \rightarrow 0$

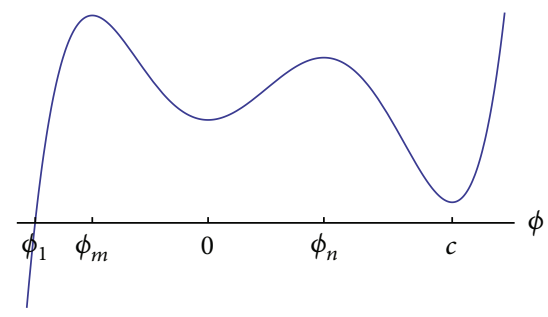

(i) $h>h_{c}$

Figure 8: The graph of $F(\phi)$ as $(1 / 5)\left(5 c-2 c^{2}\right)<c_{0}<c$.

double stumpons which contain intervals where $\phi=c$ (see Figure 1(l)). Since any countable number of wave segments can be joined together, one can get travelling waves with very strange profiles, such as the travelling waves with a fractal appearance where $\mu\left(\varphi^{-1}(c)\right)=0$ (see Figure $1(\mathrm{k})$ ). Then the proof of Theorem 11 is completed.

\section{Conclusions}

By an improved method combining some characteristics of several methods, we have obtained abundant traveling waves in the $\mathrm{mDP}$ equation. Those solutions include looped wave solutions, cusped wave solutions, peaked wave solutions, 


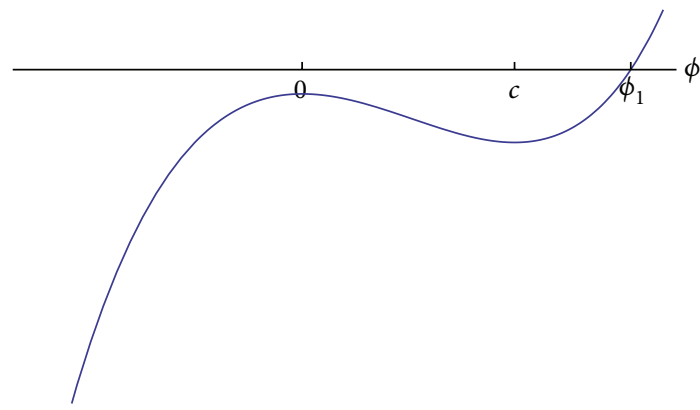

(a) $h<0$

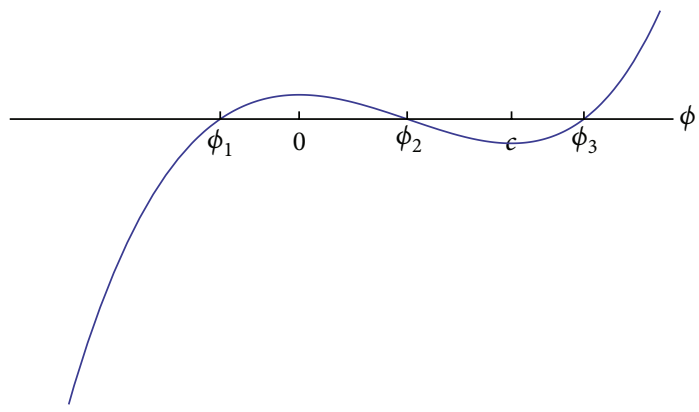

(c) $h \in\left(0, h_{c}\right)$

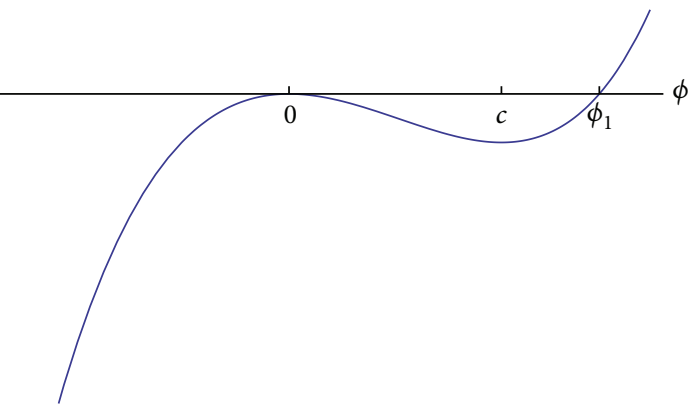

(b) $h \rightarrow 0$

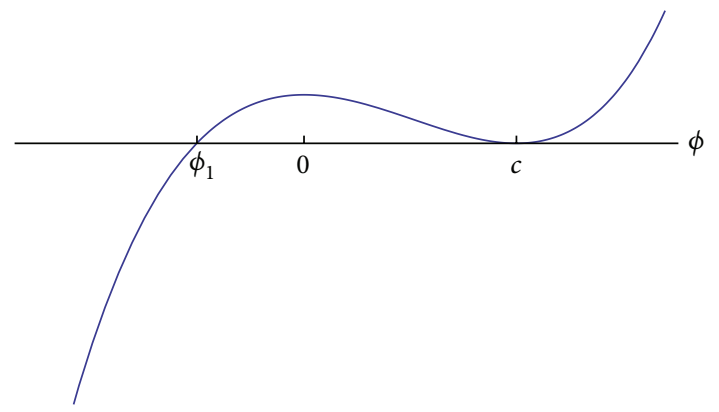

(d) $h \rightarrow h_{c}$

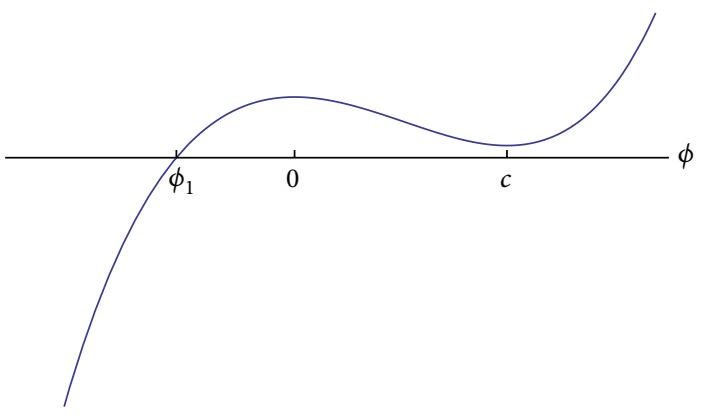

(e) $h>h_{c}$

FIGURE 9: The graph of $F(\phi)$ as $c_{0} \geq c$.

fractal-like waves, double stumpons, double kinked waves, and butterfly-like waves. Under different parametric conditions, various sufficient conditions to guarantee the existence of the above solutions are given. Our method can also be applied to other models where the location of extreme value points is determined. The limiting behavior of traveling wave solutions can also be given. Based on the study, it might be concluded that the improved method is useful and efficient. It can be widely applied to other nonlinear wave equations. Our study may be useful to further understand the role that the nonlinearly dispersive terms pay on the optical wave solutions.

\section{Conflict of Interests}

The authors declare that they have no conflict of interests regarding the publication of this paper.

\section{Acknowledgments}

This work is supported by the National Nature Science Foundation of China (no. 11101191) and it is also sponsored by the Qing Lan Project.

\section{References}

[1] A. Constantin and D. Lannes, "The hydrodynamical relevance of the Camassa-Holm and Degasperis-Procesi equations," Archive for Rational Mechanics and Analysis, vol. 192, no. 1, pp. 165-186, 2009.

[2] H. R. Dullin, G. A. Gottwald, and D. D. Holm, "An integrable shallow water equation with linear and nonlinear dispersion," Physical Review Letters, vol. 87, no. 19, Article ID 194501, 4 pages, 2001.

[3] G. M. Coclite and K. H. Karlsen, "On the well-posedness of the Degasperis-Procesi equation," Journal of Functional Analysis, vol. 233, no. 1, pp. 60-91, 2006. 
[4] F. Guo, "Global weak solutions and wave breaking phenomena to the periodic Degasperis-Procesi equation with strong dispersion," Nonlinear Analysis: Theory, Methods \& Applications, vol. 71, no. 11, pp. 5280-5295, 2009.

[5] D. Henry, "Infinite propagation speed for the DegasperisProcesi equation," Journal of Mathematical Analysis and Applications, vol. 311, no. 2, pp. 755-759, 2005.

[6] D. D. Holm and M. F. Staley, "Nonlinear balance and exchange of stability of dynamics of solitons, peakons, ramps/cliffs and leftons in a $1+1$ nonlinear evolutionary PDE," Physics Letters A, vol. 308, no. 5-6, pp. 437-444, 2003.

[7] Z. W. Lin and Y. Liu, "Stability of peakons for the DegasperisProcesi equation," Communications on Pure and Applied Mathematics, vol. 62, no. 1, pp. 151-163, 2009.

[8] H. Liu, "Wave breaking in a class of nonlocal dispersive wave equations," Journal of Nonlinear Mathematical Physics, vol. 13, no. 3, pp. 441-466, 2006.

[9] Y. Liu and Z. Y. Yin, "Global existence and blow-up phenomena for the Degasperis-Procesi equation," Communications in Mathematical Physics, vol. 267, no. 3, pp. 801-820, 2006.

[10] H. Lundmark, "Formation and dynamics of shock waves in the Degasperis-Procesi equation," Journal of Nonlinear Science, vol. 17, no. 3, pp. 169-198, 2007.

[11] B. Guo and Z. Liu, "Periodic cusp wave solutions and singlesolitons for the b-equation," Chaos, Solitons and Fractals, vol. 23, no. 4, pp. 1451-1463, 2005.

[12] W. Rui, B. He, Y. Long, and C. Chen, "The integral bifurcation method and its application for solving a family of thirdorder dispersive PDEs," Nonlinear Analysis: Theory, Methods \& Applications, vol. 69, no. 4, pp. 1256-1267, 2008.

[13] C. Chen and M. Y. Tang, "A new type of bounded waves for Degasperis-Procesi equation," Chaos, Solitons and Fractals, vol. 27, no. 3, pp. 698-704, 2006.

[14] V. O. Vakhnenko and E. J. Parkes, "Periodic and solitary-wave solutions of the Degasperis-Procesi equation," Chaos, Solitons \& Fractals, vol. 20, no. 5, pp. 1059-1073, 2004.

[15] J. Lenells, "Traveling wave solutions of the Degasperis-Procesi equation," Journal of Mathematical Analysis and Applications, vol. 306, no. 1, pp. 72-82, 2005.

[16] A. M. Wazwaz, "New solitary wave solutions to the modified forms of Degasperis-Procesi and Camassa-Holm equations," Applied Mathematics and Computation, vol. 186, no. 1, pp. 130141, 2007.

[17] H. Ma, Y. Yu, and D. Ge, "New exact traveling wave solutions for the modified form of Degasperis-Procesi equation," Applied Mathematics and Computation, vol. 203, no. 2, pp. 792-798, 2008.

[18] W. Rui, B. He, S. Xie, and Y. Long, "Application of the integral bifurcation method for solving modified Camassa-Holm and Degasperis-Procesi equations," Nonlinear Analysis: Theory, Methods and Applications, vol. 71, no. 7-8, pp. 3459-3470, 2009.

[19] Z. Liu and Z. Ouyang, "A note on solitary waves for modified forms of Camassa-Holm and Degasperis-Procesi equations," Physics Letters. A, vol. 366, no. 4-5, pp. 377-381, 2007. 


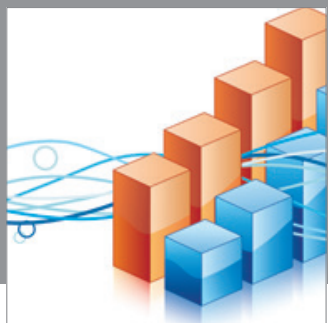

Advances in

Operations Research

mansans

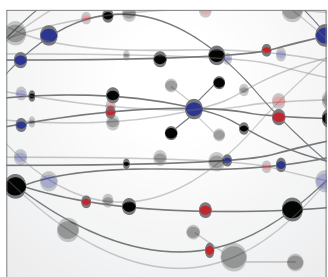

The Scientific World Journal
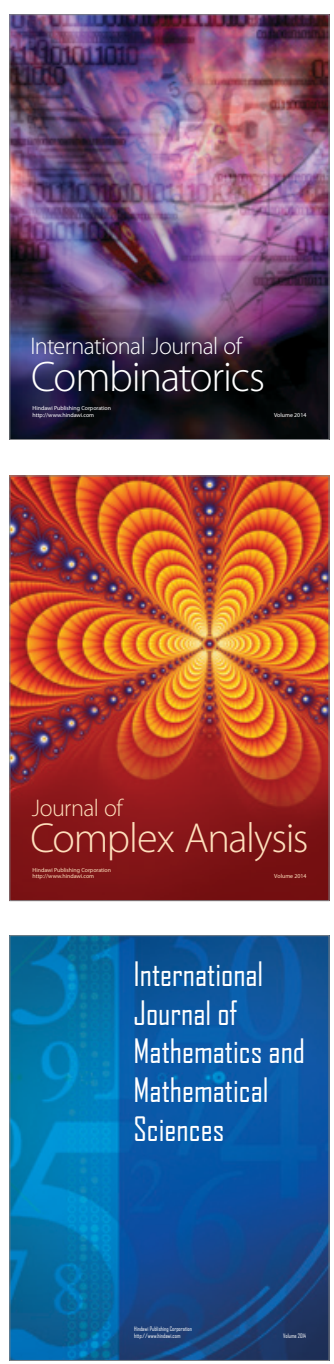
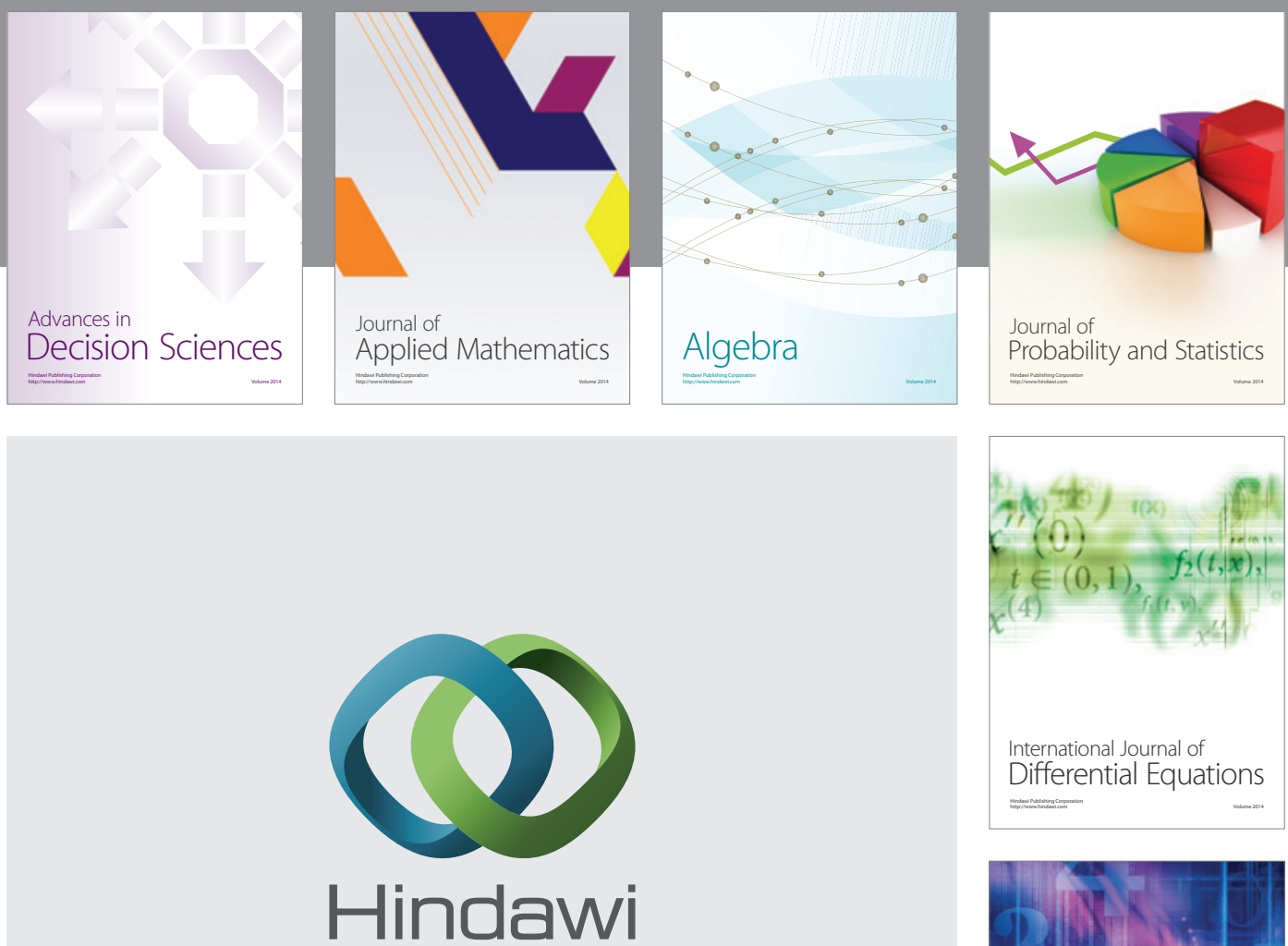

Submit your manuscripts at http://www.hindawi.com
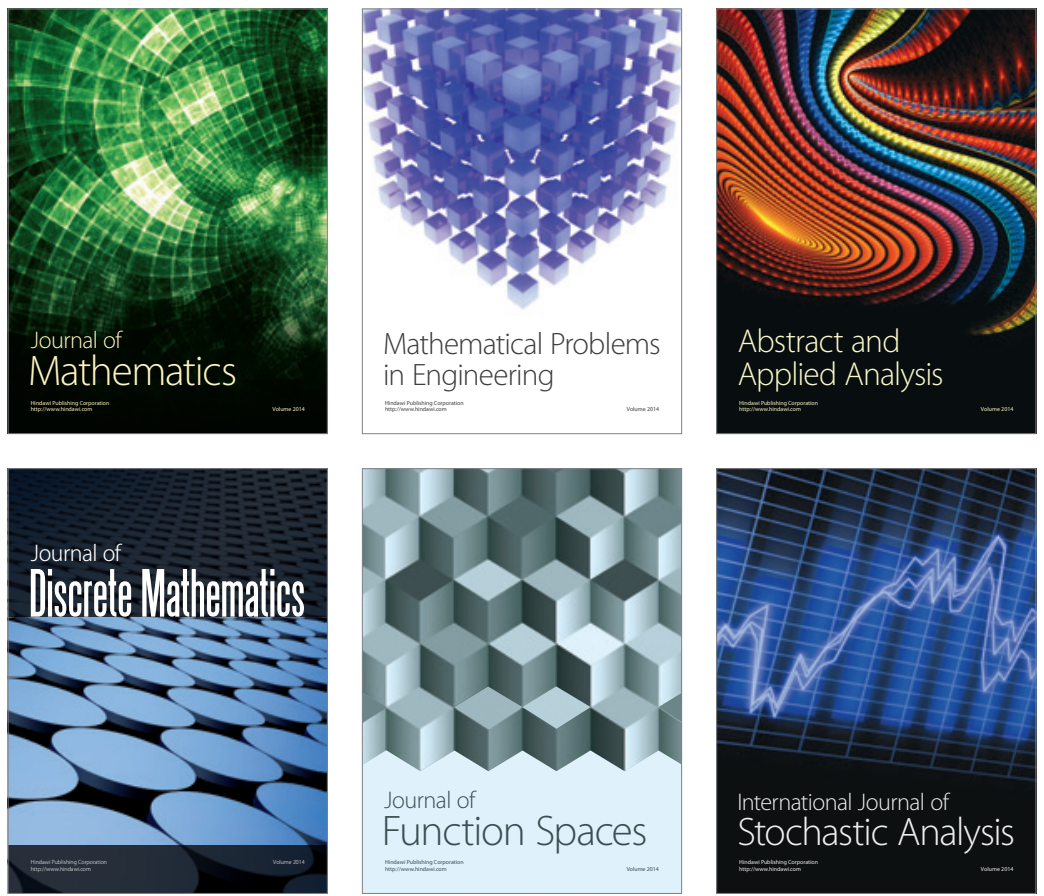

Journal of

Function Spaces

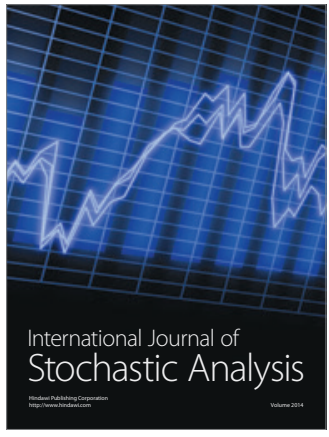

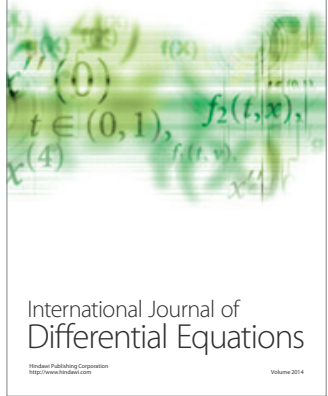
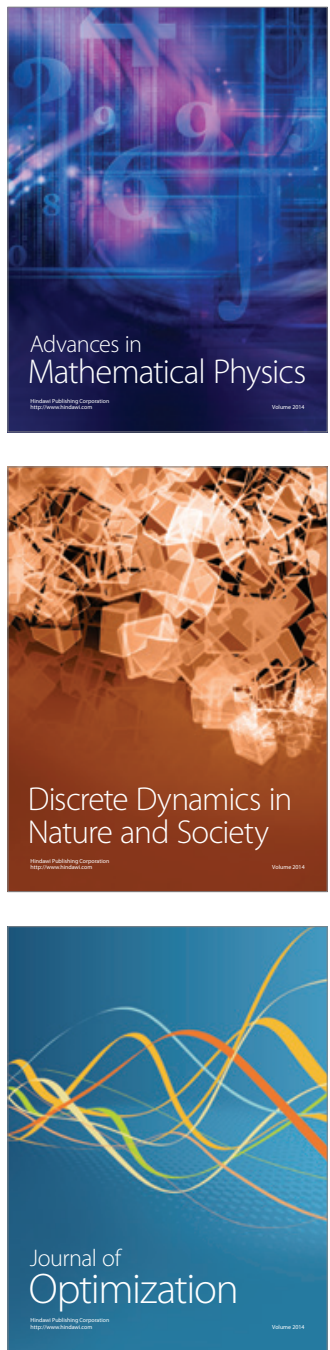